\title{
Real-World Effectiveness of Sodium Glucose Co-Transporter-2 Inhibitors in Japanese Patients with Diabetes Mellitus
}

\author{
Yuichiro Ito · James Van Schyndle - Takuya Nishimura • \\ Toshifumi Sugitani $\cdot$ Tomomi Kimura (1)
}

Received: August 29, 2019 / Published online: October 15, 2019

(C) The Author(s) 2019

\begin{abstract}
Introduction: Sodium glucose co-transporter-2 inhibitors (SGLT2i) have been on the market for 5 years in Japan. We explored the real-world effectiveness of SGLT2i in Japan.

Methods: We retrospectively analyzed two large Japanese administrative databases from JMDC Inc.: insurance-dataset (I-dataset) and Medical Data Vision Co. Ltd. [hospital-dataset (H-dataset)]. Patients who newly started SGLT2i or other oral antidiabetic drugs (OADs) between 1 April 2014 and 31 March 2016 were selected for this analysis and followed for 1 year from the index date. Changes in glycated hemoglobin (HbA1c), body mass index (BMI), and estimated glomerular filtration rate (eGFR) were evaluated during the 1-year period.
\end{abstract}

Enhanced Digital Features To view enhanced digital features for this article go to https://doi.org/10.6084/ m9.figshare.9918647.

Electronic supplementary material The online version of this article (https://doi.org/10.1007/s13300019-00708-w) contains supplementary material, which is available to authorized users.

Y. Ito · T. Nishimura · T. Sugitani · T. Kimura ( $₫)$

Astellas Pharma Inc., Tokyo, Japan

e-mail: tomomi.kimura@astellas.com

J. Van Schyndle

Astellas US, LLC., Northbrook, IL, USA
Results: A total of 127,961 patients in the H-dataset and 26,436 in the I-dataset were included in this analysis. Baseline HbA1c, BMI, and eGFR levels tended to be higher in SGLT2i users than in other OAD cohorts. After 1 year, $44.3 \%$ (I-dataset) and $53.3 \%$ (H-dataset) of SGLT2i users and 33.0-44.2\% (I-dataset) and 47.0-58.1\% (H-dataset) of other users were still on their medications. The mean HbA1c level decreased by -0.7 to $-0.9 \%$ in SGLT2i users versus -0.4 to $-1.5 \%$ in the other cohorts. The mean BMI decreased by $-0.8 \mathrm{~kg} / \mathrm{m}^{2}$ in SGLT2i users whereas the change in other cohorts was -0.5 to $0.4 \mathrm{~kg} / \mathrm{m}^{2}$. No clinically relevant changes in eGFR were observed over the period. Conclusion: This study showed that around half of the SGLT2i users were still on medication after 1 year from treatment initiation. Initiation of SGLT2i was associated with improvement in HbA1c and BMI, with no abnormal changes in renal function observed in the first year following treatment. These findings support the results from clinical trials and will expand the existing evidence of SGLT2i use in real-life practice in Japan.

Funding: Astellas Pharma Inc., Tokyo, Japan.

Keywords: Administrative databases; BMI; Effectiveness; Glycemic control; HbA1c; Japan; Real-world; Renal function; SGLT2i 


\section{INTRODUCTION}

Uncontrolled hyperglycemia can lead to a host of micro- and macrovascular complications in patients with diabetes. The main goals of diabetes treatment are to achieve optimal glycemic control and reduce the risks of these complications [1]. Patients with type 2 diabetes mellitus (T2DM) are often overweight or obese, and they are at an increased risk for cardiovascular (CV) comorbidities. In addition to adequate glycemic control, weight management is recognized as an important aspect of diabetes management in patients with T2DM [1]. Patients with T2DM also have increased risk of adverse renal outcomes [2]. Hence, antidiabetic drugs that offer good glycemic control, with the additional benefits of weight loss and renoprotection, would be desirable treatment options for patients with T2DM.

Between April 2014 and February 2015, six sodium glucose co-transporter-2 inhibitors (SGLT2i), ipragliflozin, dapagliflozin, luseogliflozin, tofogliflozin, canagliflozin, and empagliflozin, were approved in Japan for the treatment of patients with T2DM [3-11]. SGLT2i are a class of oral antidiabetic drug (OAD), which lowers blood glucose levels by inhibiting glucose reabsorption within the kidneys, resulting in urinary glucose excretion and consequent loss of calories [12]. The efficacy and safety of SGLT2i in Japanese patients with T2DM have been well established in clinical trials [3-11]. Results from clinical trials showed that SGLT2i reduced glycated hemoglobin (HbA1c), with the added benefit of weight loss when used either alone or in combination other OADs [3-11]. Recently, SGLT2 inhibition has been associated with additional renoprotective effects in patients with T2DM [13-17].

In Japan, a few studies have evaluated the clinical effectiveness of SGLT2i in patients with T2DM in the real-world setting [18-21]. These studies examined selected SGLT2i compounds [18-21] and were mostly conducted only in elderly patients [19-21]. More data on the clinical effectiveness of SGLT2i treatment in Japanese patients are needed to guide real-life practice and treatment planning. Previously, we reported the characteristics of SGLT2i users in the first 3 years following the launch of SGLT2i in Japan, using data obtained from three large Japan-specific administrative databases [22]. Here, we further examined the changes in clinical measures [HbA1c, body mass index (BMI), estimated glomerular filtration rate (eGFR)] in the first year after treatment initiation among SGLT2i users to expand on the limited data in these areas.

\section{METHODS}

This was a retrospective study using two administrative databases from Japan. The protocol for this study was approved by the Medical Affairs Japan Protocol Review Committee on 22 June 2017 prior to study commencement. As the study only used existing data in the databases, written informed consent was not required. This study was conducted in compliance with the guidelines for Good Pharmacoepidemiology Practice [23]. Clinical trial registration was not required for this study because it was not a prospective study and did not involve any intervention.

\section{Data Sources}

In this analysis, two independent databases were used: a hospital-based database provided by Medical Data Vision Co. Ltd. [24] (H-dataset) and an insurance claims database, linked to annual health checkup data provided by JMDC Inc. [25] (I-dataset). The H-dataset contained Diagnosis Procedure Combination (DPC) data and transaction level billing data for $>17$ million outpatients and inpatients from 287 DPC hospitals nationwide (at the time of the study). Approximately $10 \%$ of the member hospitals also provided selected blood test results (e.g., $\mathrm{HbA1c}$, serum creatinine). The $\mathrm{H}$-dataset covered all age groups and captured a larger number of patients. However, it has limited followup ability. The I-dataset contained medical and prescription claims of 3.8 million employees and their dependents, and annual health checkup data were provided for approximately 2 million of them (at the time of the study). The 
I-dataset has continuous follow-up ability regardless of the medical institution; however, subjects were mostly aged $\leq 65$ years, and no subjects aged $\geq 75$ years were included.

\section{Study Population}

The base study cohorts were selected as described elsewhere [22]. For this analysis, eligible patients were further selected. Patients aged $>18$ years as of 1 April 2014 were eligible. The index date was defined as the earliest prescription date of an OAD [SGLT2i, alpha-glucosidase inhibitors (alpha-GI), biguanides (BG), dipeptidyl peptidase-4 inhibitors (DPP-4i), glinides, sulfonylureas (SU), and thiazolidinediones (TZD)] recorded between 1 April 2014 and 31 March 2016; this had to be preceded by at least 6 months of no use of the index OAD. All SGLT2i new users were selected, and the rest were then allocated to the other OAD new user cohorts (by class). The cohorts were followed for 1 year from the index date. Patients who started $\geq 2$ OADs on the index date were excluded. The code list has been described previously [22]. Patients with T2DM were selected by excluding patients diagnosed as "diabetes otherwise specified." For the I-dataset only, patients who had $<6$ month enrollment prior to the index date were excluded from the study cohort. Patients who were hospitalized at the index date were excluded from the analysis.

Patients were then divided into sub-cohorts according to the availability of baseline HbA1c, BMI, or eGFR values (with an allowance of \pm 30 days). The I-dataset had all three clinical measures, while in the H-dataset, the BMI value was only available from inpatients and therefore was not used in this analysis. If multiple values were available within this period, the closest one to the index date was chosen.

\section{Study Assessments}

Patients in each OAD cohort were first evaluated for continued use of the index medication every 90 days: month 3 (M3), month 6 (M6), month 9 (M9), and month 12 (M12). For the I-dataset, only the M12 evaluation point was used because laboratory results were obtained from annual health checkup data. Patients were considered still on the index prescription if the days of supply encompassed each evaluation time point (i.e., M3, M6, M9, and M12). Continued use was assessed only among those still on medication at the previous evaluation point. For example, continued use at M6 was assessed only among those continuing at M3. Continuation at M9 and M12 was determined in a similar manner.

At each 90-day evaluation point, patients in each sub-cohort (HbA1c, BMI, and eGFR) were evaluated for change from baseline in HbA1c, BMI, and eGFR by index OADs if they met the criteria for continued use at each evaluation point and had at least one HbA1c, eGFR, or BMI value at each evaluation point (with an allowance of \pm 30 days). If multiple data values were available within this period, the closest one to each evaluation point was chosen.

\section{Statistical Analysis}

The data in each dataset were analyzed separately (no pooled assessment). Baseline and change from baseline in HbA1c, BMI, and eGFR values at each evaluation point were descriptively summarized. HbA1c values were also categorized as: $<7.0 \%, 7.0-<7.5 \%, 7.5-<8.0 \%$, $8.0-<8.5 \%$, and $\geq 8.5 \%$. eGFR values were categorized as $\geq 60,30-<60$, and $<30 \mathrm{ml} / \mathrm{min} /$ $1.73 \mathrm{~m}^{2}$, according to eGFR categories G1-G2, G3, and G4 or above, respectively, in chronic kidney disease. All analyses were performed using SAS ${ }^{\circledR}$ Studio Release 3.5 (SAS Institute Inc., Cary, NC, USA).

\section{RESULTS}

\section{Patient Demographics and Baseline Characteristics}

In total, 127,961 patients in the $\mathrm{H}$-dataset and 26,436 patients in the I-dataset were evaluated. Among them, 12,654 and 10,987 patients were included in the HbA1c and eGFR sub-cohorts, respectively, in the H-dataset and 1442, 1641, 
and 799 patients were included in the Hb1Ac, BMI, and eGFR sub-cohorts, respectively, in the I-dataset. The number of patients at baseline and each evaluation point is shown in Table S1. After 1 year, $44.3 \%$ (I-dataset) and 53.3\% (Hdataset) of SGLT2i users and 33.0-44.2\% (Idataset) and $47.0-58.1 \%$ (H-dataset) of other users were still receiving their index prescriptions.

The baseline characteristics of each sub-cohort are presented in Tables 1, 2, and 3. Patients who did not have baseline HbA1c, BMI, or eGFR values had a demographic profile fairly similar to those who had baseline values (Table S2-S4).

The mean baseline HbA1c level tended to be higher in SGLT2i users than in other OAD cohorts in the H-dataset; it was comparable among all OAD cohorts (except for SU users and $\alpha$-GI users) in the I-dataset (Table 1). SGLT2i users had the highest proportion of patients who had $\mathrm{HbA1c} \geq 8.5 \%$ at baseline in the H-dataset (Fig. 1a). The proportion was fairly similar across all OAD cohorts (apart from $\alpha$-GI users) in the I-dataset (Fig. 1b). The mean baseline BMI tended to be higher in SGLT2i users compared with other OAD cohorts (Table 2). The mean baseline eGFR values tended to be higher in SGLT2i users than in other OAD cohorts (except for BG users) in the H-dataset; it was broadly similar across the OAD cohorts in the I-dataset (Table 3). SGLT2i users and BG users had the highest proportion of patients with eGFR $>60 \mathrm{ml} / \mathrm{min} / 1.73 \mathrm{~m}^{2}$ at baseline (83.1\% and $83.3 \%$, respectively, vs. $56.7-70.5 \%$ in other cohorts) in the H-dataset. The proportion was comparable between SGLT2i and other OAD cohorts $(88.8 \%$ and $80.4-100.0 \%$, respectively) in the I-dataset.

\section{Change from Baseline in Clinical Measures}

A notable increase in the proportion of patients who had HbA1c level $<7 \%$ was observed in all OAD cohorts at 12 months (Fig. 1). The increase among SGLT2i users (19.6-22.6\%) was broadly similar to the other OAD cohorts [ $\alpha-\mathrm{GI}$ (13.8-21.1\%), glinide (12-27.4\%), SU (14.4-36.8\%), and TZD (12.4-19.6\%)], except for BG users (26.5-34.9\%) and DPP-4i users
(26.1-32.2\%). HbA1c levels decreased from baseline in each OAD cohort in the datasets over the 12-month period (Fig. 2). The reduction in HbA1c level at 12 months was slightly greater in SGLT2i users, BG users, and DPP-4i users than in other OAD cohorts in the $\mathrm{H}$-dataset; it was comparable across all OAD cohorts (except for DPP-4i users and SU users) in the I-dataset.

Figure 3 illustrates the change in BMI at 12 months after treatment initiation by index OAD class. At 12 months, BMI decreased in SGLT2i users, $\alpha$-GI users, and BG users, with the greatest reduction noted in SGLT2i users, whereas minimal change in BMI or increased BMI was noted in the other cohorts. Change in eGFR over the first 12 months following treatment initiation by index OAD class is shown in Table S5. No clinically relevant changes in eGFR were noted in all OAD cohorts in the datasets over the 12-month period. Figure 4 illustrates the proportion of patients at each eGFR category at baseline and 12 months following treatment initiation. No marked changes in the proportion of patients in each eGFR category were observed across all OAD cohorts at 12 months.

\section{DISCUSSION}

The results from this analysis of data obtained from two large administrative databases in Japan showed that around half of the SGLT2i users were still receiving their prescribed medications at 1 year following treatment initiation. In patients who continued SGLT2i treatment, HbA1c and BMI decreased substantially from baseline at 1 year following treatment. No substantial changes in renal function were noted.

As we previously reported, SGLT2i users were younger than other OAD users [22]. Because the I-dataset does not contain data for patients $>$ 75 years old, a relatively larger proportion of patients received SGLT2 $\mathrm{i}$ in the I-dataset $(22.3-25.9 \%)$ than in the $\mathrm{H}$-dataset (11.0-11.5\%). In this study, $44.3 \%$ and $53.3 \%$ of SGLT2i users in the I-dataset and H-dataset, respectively, were still receiving their prescriptions at 1 year following initiation. Although we 


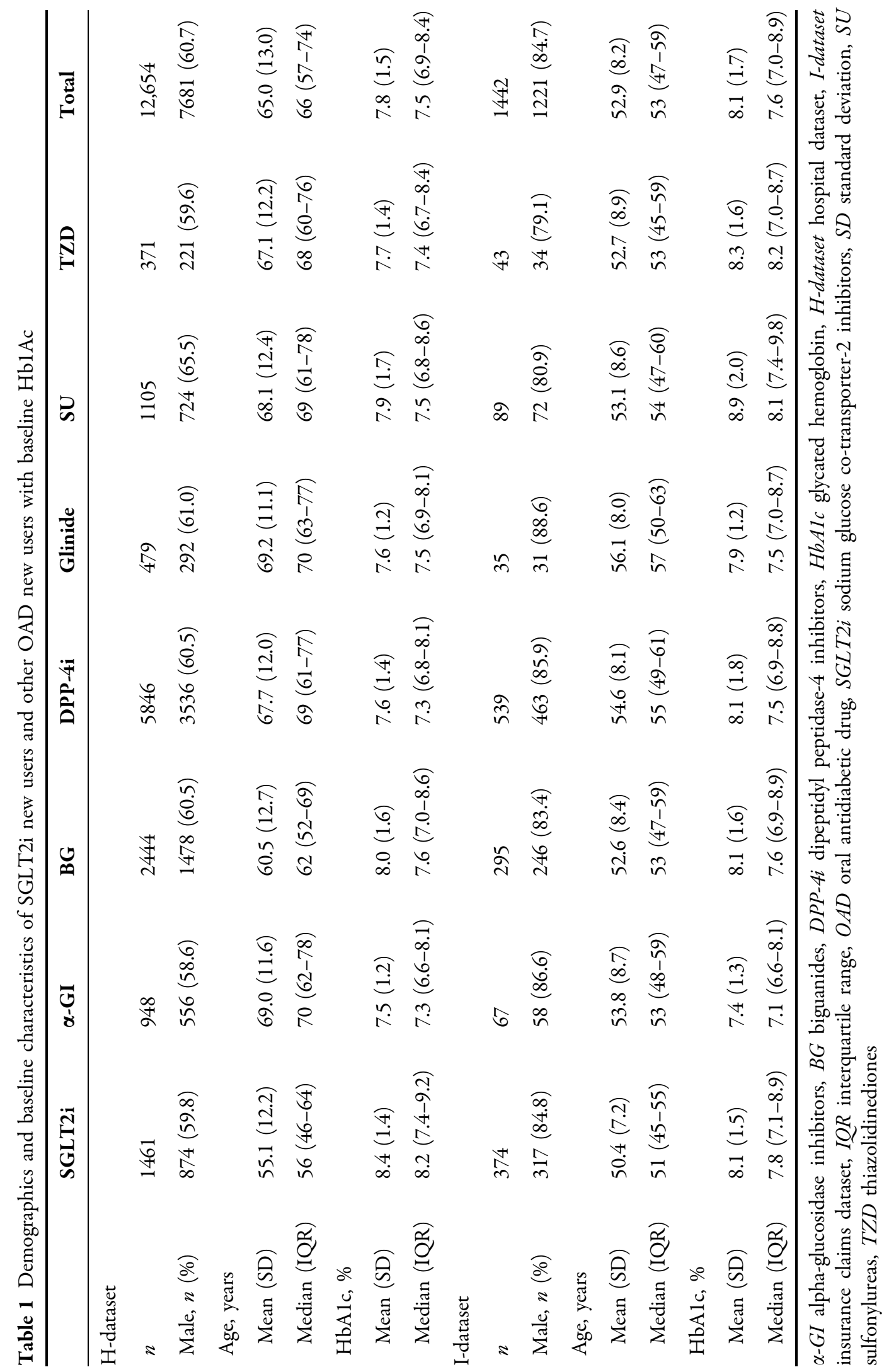




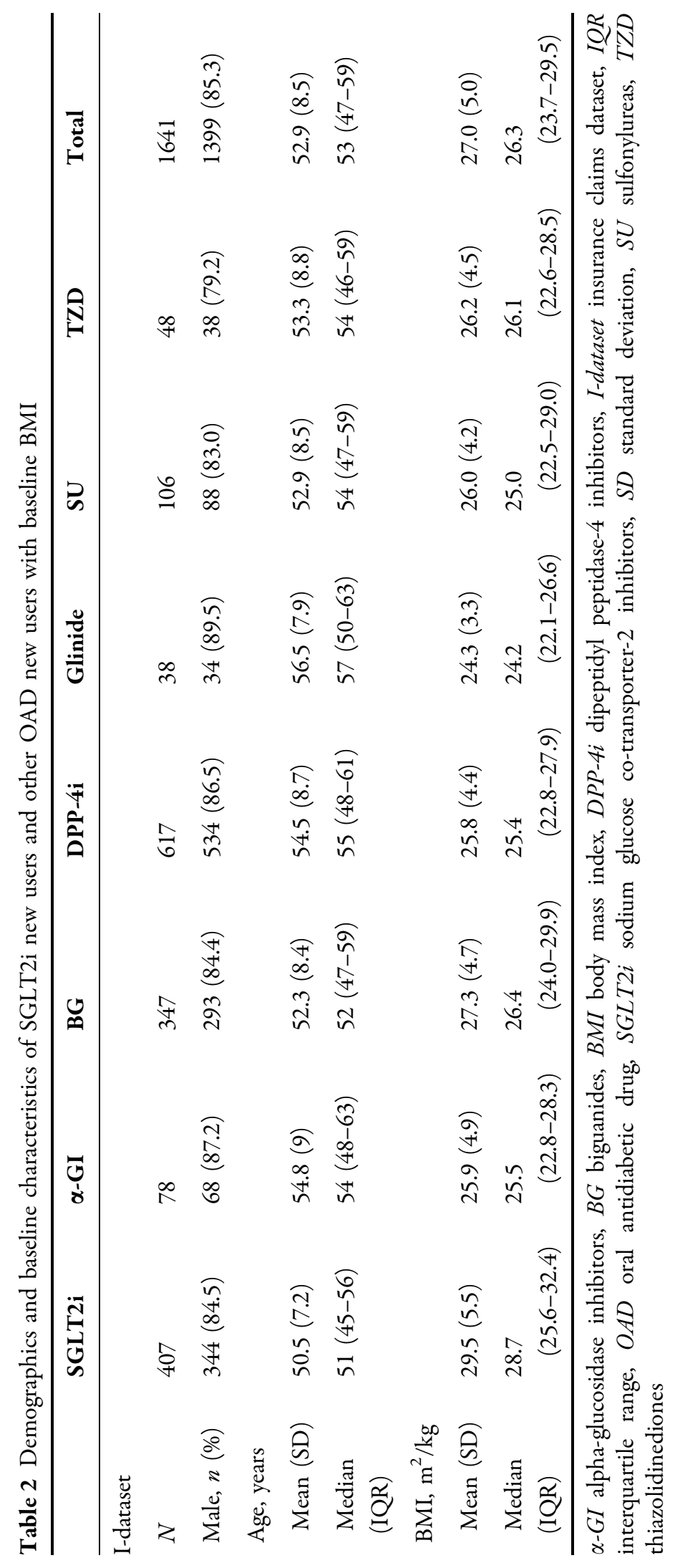




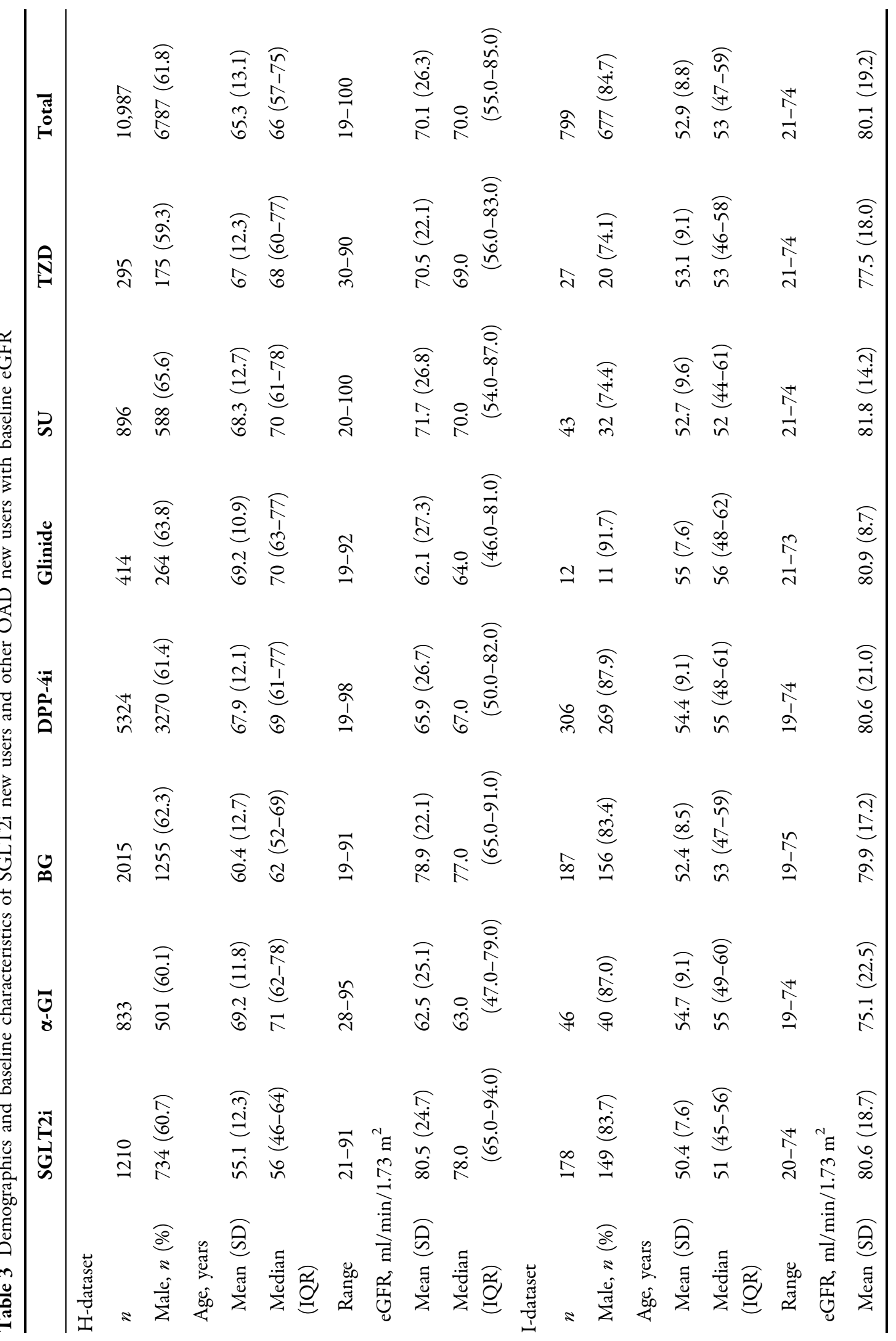




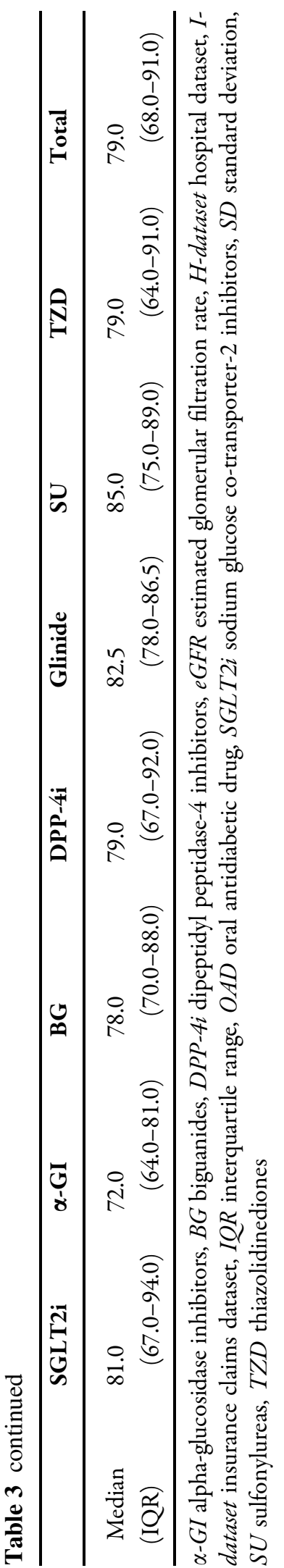

did not perform persistence analysis (e.g., time to discontinuation), this broadly concurs with the results of a recent study using the same databases, which reported 12-month persistence of SGLT2i treatment within the ranges of $53.5-62.8 \%$ in the insurance claims database and $63.4-66.4 \%$ in the hospital-based database [26].

The improvements in clinical measures observed in SGLT2i users in our study are broadly consistent with the results of clinical trials of SGLT2i in Japanese patients with T2DM [4, 7-11]. SGLT2i treatment, either alone or in combination with other OADs, was associated with $\mathrm{HbA1c}$ reductions in the range of -0.5 to $-1.3 \%$ at the end of the 1-year observation period in the clinical trials [4, 7-11], and our study found that SGLT2i users had HbA1c reduction of -0.7 to $-0.9 \%$. Also, in the clinical trials, patients receiving SGLT2i as a monotherapy or as an add-on to other OADs for a year had weight losses in the range of 2.0-5.5 kg [4, 7-9]. Our datasets did not have data on weight, but we found a substantial reduction in BMI $\left(-0.8 \mathrm{~kg} / \mathrm{m}^{2}\right)$. No clinically relevant changes in renal function were observed in either the clinical trials [4, 7-11] or our study. Some clinical trials have demonstrated favorable effects on renal outcomes and delayed progression of kidney disease with SGLT2 inhibition [13-17]; longer follow-up of the patients may be warranted.

Our findings also concur with the results of post-marketing studies of ipragliflozin, tofogliflozin, and canagliflozin in Japanese patients with T2DM [18-21], with HbA1c reduction of $-0.5 \%$ to $-0.8 \%$, weight reductions of $-2.7 \mathrm{~kg}$ to $-2.9 \mathrm{~kg}$, and mean change in eGFR of -0.5 to $-2.3 \mathrm{ml} / \mathrm{min} / 1.73 \mathrm{~m}^{2}$ at the end of the 1-year treatment.

Our findings need to be interpreted within the limitations of this study. Some of these limitations have already been described [22]. First, effectiveness was measured only in patients who continued index medication at each evaluation point; hence, the results are subject to survival bias. Although we allowed any add-on treatment in this analysis, patients who did not respond to their treatment might have switched to other OAD. In addition, we do 

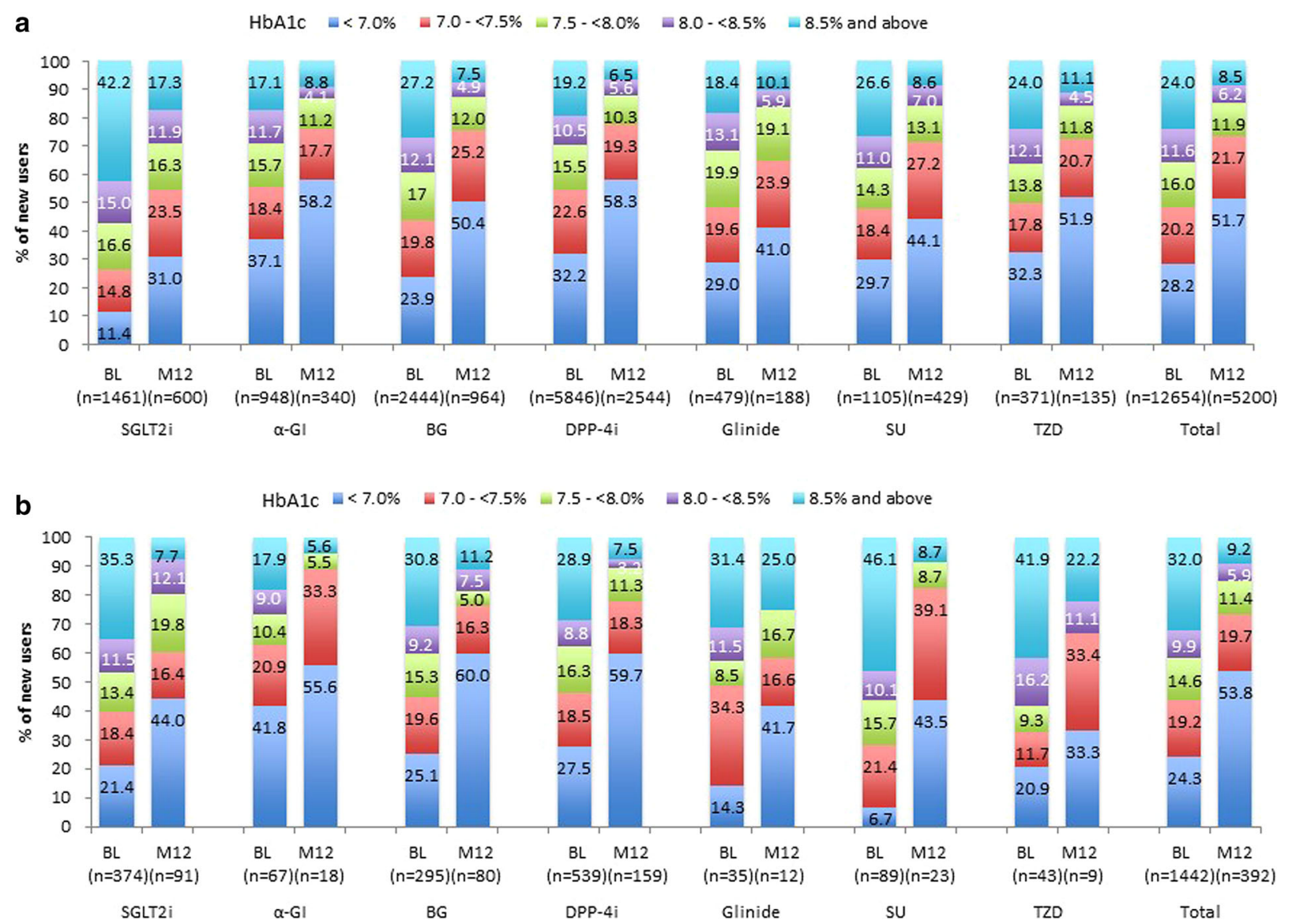

Fig. 1 Percentage of new users at each HbAlc category at baseline and 12 months by index OAD class in a $\mathrm{H}$-dataset and b I-dataset. $\alpha$-GI alpha-glucosidase inhibitors, $B G$ biguanides, $B L$ baseline, $D P P-4 i$ dipeptidyl peptidase- 4 inhibitors, $H b A 1 c$ glycated hemoglobin, $H$-dataset hospital

not know if the medications were taken by the patients as prescribed in this study. Next, inpatients were excluded from the analysis because it is likely that the "index date" was the first date patients appeared in the hospital for some other reasons (e.g., surgical operation) rather than the date they truly started their index medication. In the H-dataset, patients may have reasons to take a blood test other than diabetes control (i.e., HbA1c value), and the results may not be totally generalizable. Nevertheless, the demographic characteristics of these subsets were largely similar to patients who did not have the clinical measurements of interest. The H-dataset included only DPC hospitals, and patients may have had prescriptions from other hospitals dataset, I-dataset insurance claims dataset, M12 12 months, $O A D s$ oral antidiabetic drugs, $S G L T 2 i$ sodium glucose cotransporter-2 inhibitors, $S U$ sulfonylureas, $T Z D$ thiazolidinediones

before the index date or after discontinuation. Therefore, the index prescriptions may not be truly the first prescription for some patients, or patients may have continued the index medication elsewhere. The limitations of the $\mathrm{H}$-dataset were not applicable to the I-dataset, but the latter lacked elderly patients.

\section{CONCLUSIONS}

This study showed the effectiveness of SGLT2i in the real-world setting in Japan. These findings support and add to the current body of evidence in Japanese patients and can inform 

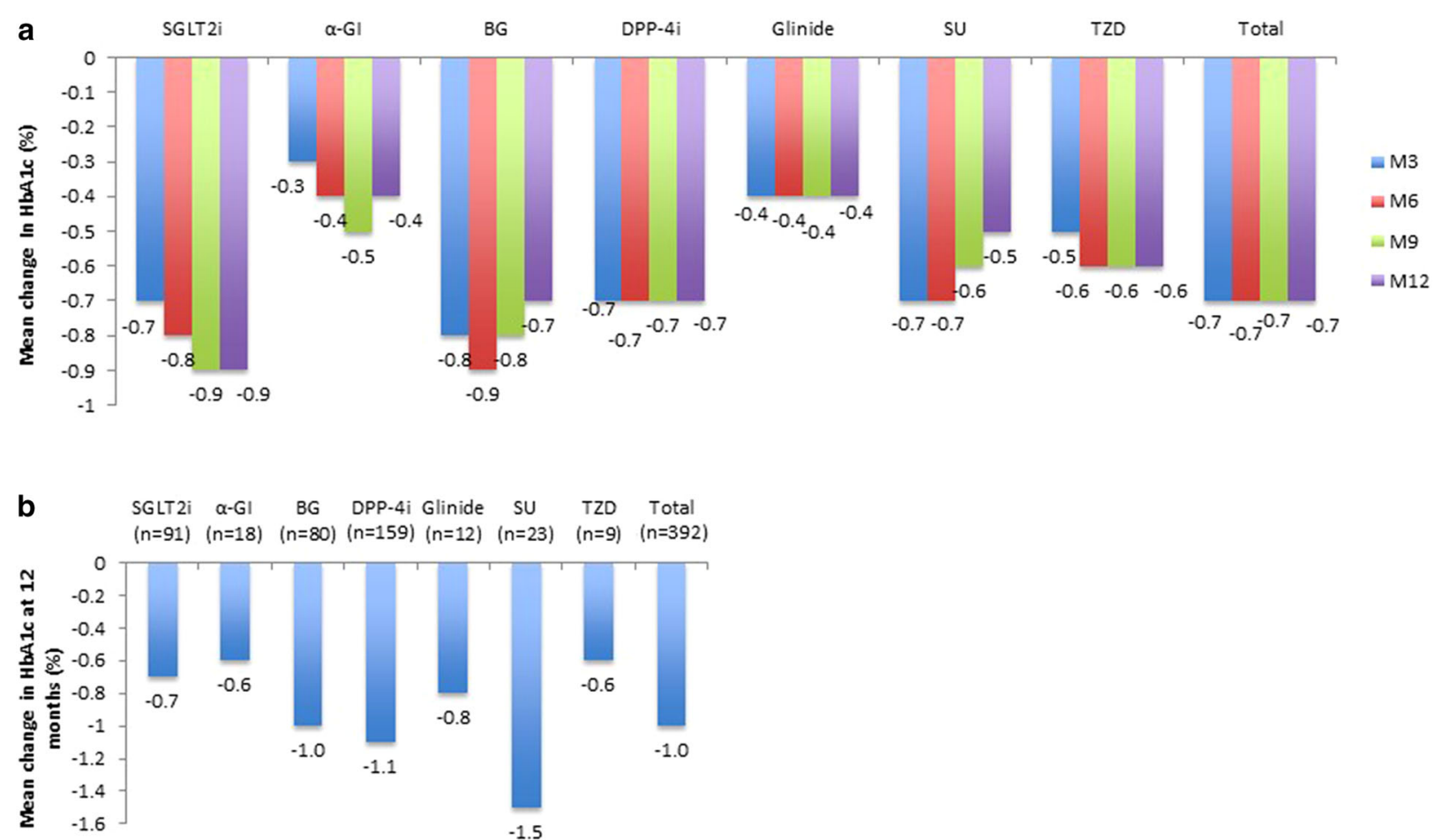

Fig. 2 Change in HbAlc level from baseline in the first 12 months following treatment initiation by index OAD class in the a H-dataset and $\mathbf{b}$ I-dataset. $\alpha$-GI alphaglucosidase inhibitors, $B G$ biguanides, $D P P-4 i$ dipeptidyl peptidase-4 inhibitors, HbAIc glycated hemoglobin, $H$ -

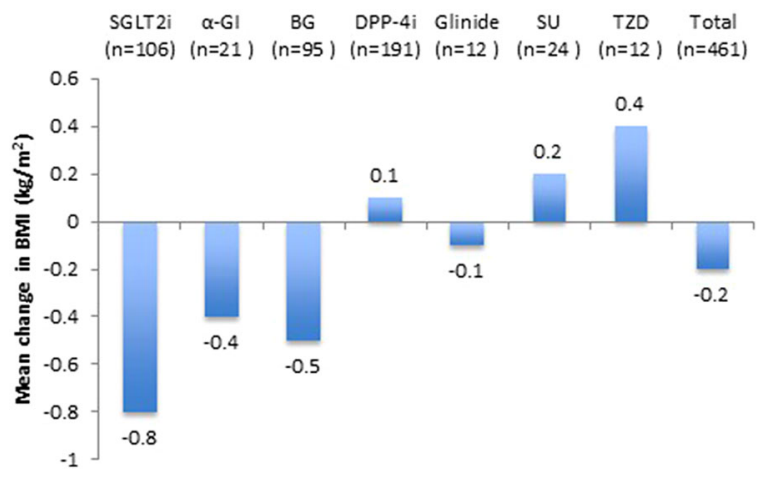

Fig. 3 Change in BMI from baseline at 12 months following treatment initiation by index OAD class (Idataset). $\alpha$ - $G I$ alpha-glucosidase inhibitors, $B G$ biguanides, $B M I$ body mass index, DPP-4i dipeptidyl peptidase- 4 inhibitors, $H b A l c$ glycated hemoglobin, I-dataset insurance claims dataset, $O A D$ oral antidiabetic drug, $S G L T 2 i$ sodium glucose co-transporter-2 inhibitors, $S U$ sulfonylureas, $T Z D$ thiazolidinediones dataset hospital dataset, I-dataset insurance claims dataset, M3 3 months, M6 6 months, M9 9 months, M12 12 months, OADs oral antidiabetic drugs, SGLT2i sodium glucose co-transporter-2 inhibitors, $S U$ sulfonylureas, $T Z D$ thiazolidinediones

the use of SGLT2 $i$ for the treatment of diabetes in real-life practice.

\section{ACKNOWLEDGEMENTS}

Funding. Sponsorship for this study and article processing charges were funded by Astellas Pharma Inc., Tokyo, Japan. All authors had access to all of the results in this study and take complete responsibility for accuracy of the data analysis.

Medical Writing and Editorial Assistance. Medical writing and editorial assistance were provided by Tech Observer Asia Pacific Pte Ltd., Singapore. Support for this assistance was funded by Astellas Pharma Inc., Tokyo, Japan. 

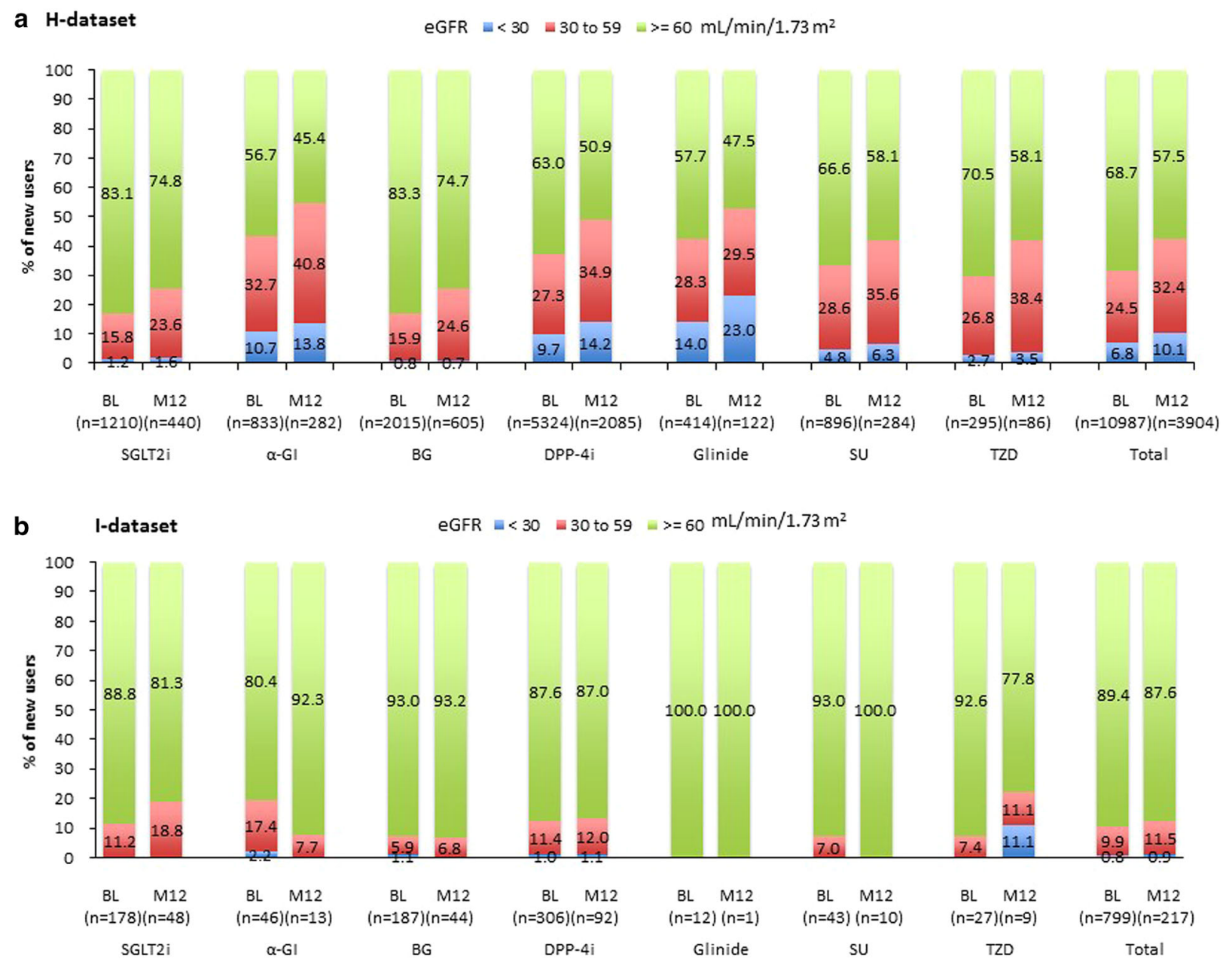

Fig. 4 Percentage of new users at each eGFR category at baseline and 12 months by index OAD class in a H-dataset and $\mathbf{b}$ I-dataset. $\alpha-G I$ alpha-glucosidase inhibitors, $B G$ biguanides, $B L$ baseline, $D P P-4 i$ dipeptidyl peptidase- 4 inhibitors, $e G F R$ estimated glomerular filtration rate, $H$ -

Authorship. All named authors meet the International Committee of Medical Journal Editors (ICMJE) criteria for authorship for this article, take responsibility for the integrity of the work as a whole, and have given their approval for this version to be published.

Disclosures. Yuichiro Ito is an employee of Astellas Pharma Inc., Tokyo, Japan. Takuya Nishimura is an employee of Astellas Pharma Inc., Tokyo, Japan. Toshifumi Sugitani is an employee of Astellas Pharma Inc., Tokyo, Japan. Tomomi Kimura is an employee of Astellas dataset hospital dataset, I-dataset insurance claims dataset, M12 12 months, OADs oral antidiabetic drugs, SGLT2i sodium glucose co-transporter-2 inhibitors, $S U$ sulfonylureas, $T Z D$ thiazolidinediones

Pharma Inc., Tokyo, Japan. James Van Schyndle is an employee of Astellas US, LLC., Northbrook, USA.

Compliance with Ethics Guidelines. The protocol for this study was approved by the Medical Affairs Japan Protocol Review Committee on 22 June 2017 prior to study commencement. As the study only used existing data in the databases, written informed consent was not required. This study was conducted in compliance with the guidelines for Good Pharmacoepidemiology Practice [23]. Clinical trial 
registration was not required for this study because it was not a prospective study and did not involve any intervention.

Data Availability. Access to anonymized individual participant data will not be provided for this study as it meets one or more of the exceptions described on http://www. clinicalstudydatarequest.com under "Sponsor Specific Details for Astellas."

Open Access. This article is distributed under the terms of the Creative Commons Attribution-NonCommercial 4.0 International License (http://creativecommons.org/licenses/ by-nc/4.0/), which permits any noncommercial use, distribution, and reproduction in any medium, provided you give appropriate credit to the original author(s) and the source, provide a link to the Creative Commons license, and indicate if changes were made.

\section{REFERENCES}

1. Haneda $M$, Noda $M$, Origasa $H$, et al. Japanese clinical practice guideline for diabetes 2016. J Diabetes Investig. 2018;9:657-97.

2. Adler AI, Stevens RJ, Manley SE, Bilous RW, Cull CA, Holman RR. Development and progression of nephropathy in type 2 diabetes: the United Kingdom Prospective Diabetes Study (UKPDS 64). Kidney Int. 2003;63:225-32. https://doi.org/10.1046/j. 523-755.2003.00712.x.

3. Kashiwagi A, Kazuta K, Yoshida S, Nagase I. Randomized, placebo-controlled, double-blind glycemic control trial of novel sodium-dependent glucose cotransporter 2 inhibitor ipragliflozin in Japanese patients with type 2 diabetes mellitus. J Diabetes Investig. 2014;5:382-91.

4. Kaku K, Maegawa H, Tanizawa Y, et al. Dapagliflozin as monotherapy or combination therapy in Japanese patients with type 2 diabetes: an open-label study. Diabetes Ther. 2014;5:415-33.

5. Ikeda S, Takano Y, Cynshi O, et al. A novel and selective sodium-glucose cotransporter-2 inhibitor, tofogliflozin, improves glycaemic control and lowers body weight in patients with type 2 diabetes mellitus. Diabetes Obes Metab. 2015;17:984-93.
6. Seino Y, Sasaki T, Fukatsu A, Ubukata M, Sakai S, Samukawa Y. Efficacy and safety of luseogliflozin as monotherapy in Japanese patients with type 2 diabetes mellitus: a randomized, double-blind, placebo-controlled, phase 3 study. Curr Med Res Opin. 2014;30:1245-55.

7. Seino $\mathrm{Y}$, Inagaki $\mathrm{N}$, Haneda $\mathrm{M}$, et al. Efficacy and safety of luseogliflozin added to various oral antidiabetic drugs in Japanese patients with type 2 diabetes mellitus. J Diabetes Investig. 2015;6:443-53.

8. Inagaki N, Kondo K, Yoshinari T, Kuki H. Efficacy and safety of canagliflozin alone or as add-on to other oral antihyperglycemic drugs in Japanese patients with type 2 diabetes: a 52-week open-label study. J Diabetes Investig. 2015;6:210-8.

9. Kadowaki T, Haneda M, Inagaki N, et al. Efficacy and safety of empagliflozin monotherapy for 52 weeks in Japanese patients with type 2 diabetes: a randomized, double-blind, parallel-group study. Adv Ther. 2015;32:306-18.

10. Tanizawa Y, Kaku K, Araki E, et al. Long-term safety and efficacy of tofogliflozin, a selective inhibitor of sodium-glucose cotransporter 2 , as monotherapy or in combination with other oral antidiabetic agents in Japanese patients with type 2 diabetes mellitus: multicenter, open-label, randomized controlled trials. Expert Opin Pharmacother. 2014;15:749-66.

11. Araki E, Tanizawa $Y$, Tanaka $Y$, et al. Long-term treatment with empagliflozin as add-on to oral antidiabetes therapy in Japanese patients with type 2 diabetes mellitus. Diabetes Obes Metab. 2015;17:665-74.

12. Chao EC, Henry RR. SGLT2 inhibition-a novel strategy for diabetes treatment. Nat Rev Drug Discov. 2010;9:551-9.

13. Heerspink HJL, Kosiborod M, Inzucchi SE, Cherney DZI. Renoprotective effects of sodium-glucose cotransporter-2 inhibitors. Kidney Int. 2018;94:26-39. https://doi.org/10.1016/j.kint. 2017.12.027 Epub 8 May 5.

14. Wanner C, Inzucchi SE, Lachin JM, et al. Empagliflozin and progression of kidney disease in type 2 diabetes. N Engl J Med. 2016;375:323-34. https:// doi.org/10.1056/NEJMoa1515920 (Epub 2016 Jun 14).

15. $\mathrm{Xu} \mathrm{L,} \mathrm{Li} \mathrm{Y,} \mathrm{Lang} \mathrm{J,} \mathrm{et} \mathrm{al.} \mathrm{Effects} \mathrm{of} \mathrm{sodium-glucose}$ co-transporter 2 (SGLT2) inhibition on renal function and albuminuria in patients with type 2 diabetes: a systematic review and meta-analysis. PeerJ. 2017;5:e3405. https://doi.org/10.7717/peerj.3405. 
16. Perkovic V, Jardine MJ, Neal B, et al. Canagliflozin and renal outcomes in type 2 diabetes and nephropathy. N Engl J Med. 2019;380:2295-306. https://doi.org/10.1056/NEJMoa1811744 Epub 2019 Apr 14.

17. Zelniker TA, Wiviott SD, Raz I, et al. SGLT2 inhibitors for primary and secondary prevention of cardiovascular and renal outcomes in type 2 diabetes: a systematic review and meta-analysis of cardiovascular outcome trials. Lancet. 2019;393:31-9. https://doi.org/10.1016/S01406736(18)32590-X Epub 2018 Nov 10.

18. Nakamura I, Maegawa H, Tobe K, Uno S. Safety and effectiveness of ipragliflozin for type 2 diabetes in Japan: 12-month interim results of the Stella-long term post-marketing surveillance study. Adv Ther. 2019;36:923-49.

19. Utsunomiya K, Shimmoto N, Senda M, et al. Safety and effectiveness of tofogliflozin in elderly Japanese patients with type 2 diabetes mellitus: a post-marketing study (J-STEP/EL Study). J Diabetes Investig. 2017;8:766-75.

20. Goda M, Yamakura T, Sasaki K, Tajima T, Ueno M. Safety and efficacy of canagliflozin in elderly patients with type 2 diabetes mellitus: a 1-year postmarketing surveillance in Japan. Curr Med Res Opin. 2018;34:319-27.

21. Yokote K, Terauchi Y, Nakamura I, Sugamori H. Real-world evidence for the safety of ipragliflozin in elderly Japanese patients with type 2 diabetes mellitus (STELLA-ELDER): final results of a post-marketing surveillance study. Expert Opin Pharmacother. 2016;17:1995-2003.

22. Ito Y, Van Schyndle J, Nishimura T, Sugitani T, Kimura T. Characteristics of patients with diabetes initiating sodium glucose co-transporter-2 inhibitors (SGLT2i): real-world results from three administrative databases in Japan. Diabetes Ther. 2019;10:549-62.

23. Public Policy Committee, International Society of Pharmacoepidemiology. Guidelines for good pharmacoepidemiology practice (GPP). Pharmacoepidemiol Drug Saf. 2016;25:2-10.

24. Introducing MDV Database: Medical Data Vision Co., Ltd. Available from: https://www.mdv.co.jp/ solution/pharmaceutical/english/. Accessed 27 April 2018.

25. Kimura S, Sato T, Ikeda S, Noda M, Nakayama T. Development of a database of health insurance claims: standardization of disease classifications and anonymous record linkage. J Epidemiol. 2010;20:413-9.

26. Nishimura R, Kato H, Kisanuki K, et al. Treatment patterns, persistence and adherence rates in patients with type 2 diabetes mellitus in Japan: a claims-based cohort study. BMJ Open. 2019;9:e025806. 